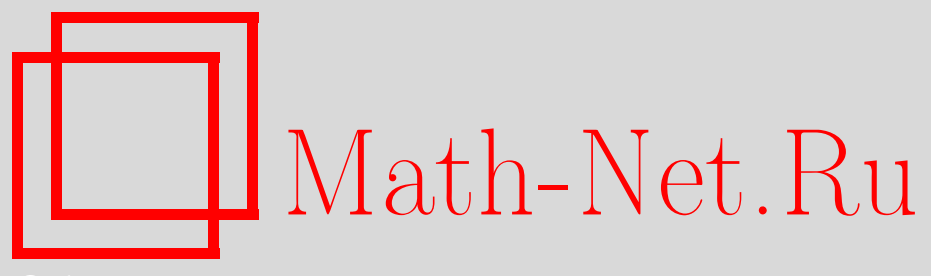

А. А. Бейлинсон, Фундаментальные решения уравнений квантовой механики как распределения и особенности решения уравнения Дирака, ТМФ, 2007, том 151, номер 2, 287-301

DOI: https://doi.org/10.4213/tmf6045

Использование Общероссийского математического портала Math-Net.Ru подразумевает, что вы прочитали и согласны с пользовательским соглашением http://www . mathnet.ru/rus/agreement

Параметры загрузки:

IP: 54.209 .52 .79

26 апреля 2023 г., 15:19:12

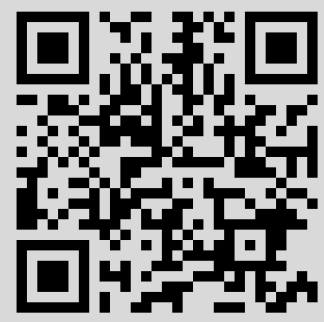




\title{
ФУНДАМЕНТАЛЬНЫЕ РЕШЕНИЯ УРАВНЕНИЙ КВАНТОВОЙ МЕХАНИКИ КАК РАСПРЕДЕЛЕНИЯ И ОСОБЕННОСТИ РЕШЕНИЯ УРАВНЕНИЯ ДИРАКА
}

\begin{abstract}
Показано, что причинные функции Грина для взаимодействующих частиц во внешних полях в релятивистской квантовой механике (для электрона Дирака) и в нерелятивистской квантовой механике могут быть получены как распределения, если использовать функции Грина свободных частиц и выбирать уравнения для соответствующих основных функций. Изучены чисто квантовые особенности решения уравнения Дирака.
\end{abstract}

Ключевые слова: причинные функции Грина, мера Винера, евклидова мера Дирака, счетная аддитивность меры, абсолютная аддитивность мер, индуцированные меры.

\section{1. ВВЕДЕНИЕ}

Мы рассмотрим построение на базе свободных частиц и взаимосвязь фундаментальных решений уравнений нерелятивистской квантовой механики и релятивистской квантовой механики в евклидовом варианте для частиц во внешних полях. В отличие от основной массы работ, восходящих к их основоположнику Фейнману (см. [1], а также [2], [3]), в основание построений положено квантовое уравнение непрерывности. В предлагаемом подходе в нерелятивистском случае с технической точки зрения все сводится к функциональной замене переменных в интеграле по мере Винера, определяющейся некоторым стохастическим уравнением, причем используется непрерывность винеровских траекторий. Следует заметить, что предлагаемый подход, опирающийся на теорему Хольмгрена-Гельфанда [4], обладает следующим преимуществом: он позволяет легко получить решение проблемы и в том случае, когда соответствующая причинная функция Грина является обобщенной функцией, т.е. когда применение метода стохастических уравнений невозможно, как это имеет место для электрона Дирака. В случае же нерелятивистской теории очевидно возможны оба пути (при этом одна и та же функция Грина строится либо как обычная гладкая функция, либо как функционал), и в работе рассматриваются оба подхода.

\footnotetext{
*Российский университет дружбы народов, Москва, Россия. E-mail: alal@m9com.ru
} 
В случае уравнения Дирака метод иллюстрируется примером решения задачи об электроне во внешнем электростатическом поле. Следует отметить, что в задачах на уравнение Дирака обнаруживается кинематическая взаимосвязь движений по ортогональным направлениям, исчезающая при переходе к квазиклассическому и нерелятивистскому пределам.

\section{2. НЕРЕЛЯТИВИСТСКИЙ СЛУЧАЙ}

Рассмотрим сначала уравнение Блоха-Шредингера в евклидовой нерелятивистской квантовой механике [5]

$$
\frac{\partial}{\partial t} Z_{t}^{v}(x)=-\widehat{H}^{v} Z_{t}^{v}(x), \quad \widehat{H}^{v}=-\frac{1}{4} \frac{\partial^{2}}{\partial x^{2}}+V(x)
$$

Здесь $\widehat{H}^{v}$ - оператор энергии в квантовой механике. Для простоты записи рассмотрим одномерный случай и будем использовать систему единиц, в которой $\hbar=1$, $m=2$.

Будем рассматривать также еще одно решение $z_{t}^{v}(x)$ этого уравнения; очевидно, $z_{-t}^{v}(x)$ удовлетворяет уравнению Блоха вспять во времени:

$$
\frac{\partial}{\partial t} z_{-t}^{v}(x)=\widehat{H}^{v} z_{-t}^{v}(x)
$$

Рассмотрим стохастическое уравнение

$$
\dot{x}(t)-\frac{1}{2} \frac{\partial \ln z_{-t}^{v}(x(t))}{\partial x(t)}=\dot{\varphi}(t),
$$

где $\varphi(t)$ - винеровский процесс [5]-[7], отвечающий свободной евклидовой квантовой частице.

Напомним, что винеровская мера процесса $\varphi(s), 0 \leqslant s \leqslant t$, есть

$$
d_{\mathrm{W}} \varphi(s)=\exp \left\{-\int_{0}^{t} \dot{\varphi}^{2}(s) d s\right\} \prod_{s=0}^{t} \frac{d \varphi(s)}{\sqrt{\pi d s}} .
$$

Эта мера счетно-аддитивна [5].

Найдем винеровскую меру траектории $x(s)$; иначе говоря, сделаем замену переменных, соответствующую уравнению (3), в мере (4). В результате получим

$$
\begin{aligned}
\exp \left\{-\int_{0}^{t}\right. & {\left.\left[\dot{x}(s)-\frac{1}{2} \frac{\partial \ln z_{-s}^{v}(x(t))}{\partial x(s)}\right]^{2} d s\right\} \times } \\
& \times \exp \left\{-\frac{1}{4} \int_{0}^{t} \frac{\partial^{2} \ln z_{-s}^{v}(x(s))}{\partial x^{2}(s)} d s\right\} \prod_{s=0}^{t} \frac{d x(s)}{\sqrt{\pi d s}}
\end{aligned}
$$

Отметим, что вторая экспонента в (5) есть якобиан (знаменатель Фредгольма) замены переменных (3), см. [6]. 
Если теперь проинтегрировать (5) по множеству траекторий с фиксированными концами $x(0)$ и $x(t)$, то получим интеграл по мере Винера

$$
\begin{gathered}
W_{t}\left(x_{0}, x_{t}\right)=\int_{C} \exp \left\{-\int_{0}^{t}\left(\left[\dot{x}(s)-\frac{1}{2} \frac{\partial \ln z_{-s}^{v}(x(s))}{\partial x(s)}\right]^{2}+\right.\right. \\
\left.\left.+\frac{1}{4} \frac{\partial^{2} \ln z_{-s}^{v}(x(s))}{\partial x^{2}(s)}\right) d s\right\} \prod_{s=0}^{t} \frac{d x(s)}{\sqrt{\pi d s}}, \\
x(0)=x_{0}, \quad x(t)=x_{t},
\end{gathered}
$$

который является фундаментальным решением уравнения Фоккера-Планка [6]

$$
\frac{\partial W}{\partial t}+\frac{1}{2} \frac{\partial}{\partial x}\left[\frac{\partial \ln z_{-t}^{v}(x)}{\partial x} W\right]=\frac{1}{4} \frac{\partial^{2} W}{\partial x^{2}} .
$$

Выполним теперь интегрирование в показателе экспоненты под знаком винеровского интеграла в (6), выделяя полную производную по времени от $\ln z_{-s}^{v}(x(s))$ и тождественно преобразуя оставшееся выражение с использованием (2); в результате получим

$$
\begin{gathered}
W_{t}\left(x_{0}, x_{t}\right)=\frac{z_{-t}^{v}\left(x_{t}\right)}{z_{0}^{v}\left(x_{0}\right)} \int_{C} \exp \left\{-\int_{0}^{t}\left[\dot{x}^{2}(s)+V(x(s))\right] d s\right\} \prod_{s=0}^{t} \frac{d x(s)}{\sqrt{\pi d s}} \\
x(0)=x_{0}, \quad x(t)=x_{t} .
\end{gathered}
$$

Так как согласно формуле Каца-Фейнмана [5] функциональный интеграл в (8) является фундаментальным решением $Z_{t}^{v}\left(x_{0}, x_{t}\right)$ уравнения $(1)$, то мы имеем

$$
W_{t}\left(x_{0}, x_{t}\right)=\frac{z_{-t}^{v}\left(x_{t}\right)}{z_{0}^{v}\left(x_{0}\right)} Z_{t}^{v}\left(x_{0}, x_{t}\right),
$$

и эта формула представляет собой утверждение известной теоремы о факторизации фундаментального решения уравнения Фоккера-Планка (7) [7]. Таким образом, замена переменных (3) в мере Винера привела к изменению оператора энергии и учету внешнего поля в уравнениях (1) и (2), которым удовлетворяют сомножители в (9). При этом информация об этом внешнем поле содержится в стохастическом уравнении (3) и определяется конкретным решением $z_{-t}^{v}(x)$ уравнения (2). Поэтому введение этого заданного внешнего поля очевидно можно осуществить не одной, а многими заменами переменных, каждая из которых определяется фиксированным решением уравнения (2). При этом изменяется только дробь в правой части равенства $(9)$, а $Z_{t}^{v}\left(x_{0}, x_{t}\right)$ остается неизменной. Еще раз подчеркнем: $Z_{t}^{v}\left(x_{0}, x_{t}\right)$ является фундаментальным решением уравнения Блоха с тем же оператором Гамильтона, что у $z_{-t}^{v}\left(x_{t}\right)$.

Методом функциональной замены переменных также легко учесть взаимодействие квантовых частиц. В простейшем случае двух одномерных частиц, если ему отвечает уравнение Блоха вспять во времени,

$$
\frac{\partial}{\partial t} z_{-t}^{v}\left(x_{1}, x_{2}\right)=\widehat{H}^{v} z_{-t}^{v}\left(x_{1}, x_{2}\right), \quad \widehat{H}^{v}=-\frac{1}{4} \Delta+V\left(x_{1}, x_{2}\right),
$$


то замена переменных, соответствующая системе стохастических уравнений

$$
\begin{aligned}
& \dot{\varphi}_{1}(t)=\dot{x}_{1}(t)-\frac{1}{2} \frac{\partial}{\partial x_{1}} \ln z_{-t}^{v}\left[x_{1}(t), x_{2}(t)\right] \\
& \dot{\varphi}_{2}(t)=\dot{x}_{2}(t)-\frac{1}{2} \frac{\partial}{\partial x_{2}} \ln z_{-t}^{v}\left[x_{1}(t), x_{2}(t)\right]
\end{aligned}
$$

где $\varphi_{1}(t), \varphi_{2}(t)$ - независимые винеровские процессы, решает задачу. При этом возникает следующий вариант теоремы о факторизации фундаментального решения уравнения Фоккера-Планка, отвечающего этой системе стохастических уравнений [6], [7]:

$$
W_{t}\left(x_{1_{0}}, x_{2_{0}} ; x_{1}, x_{2}\right)=\frac{z_{-t}^{v}\left(x_{1}, x_{2}\right)}{z_{0}^{v}\left(x_{1_{0}}, x_{2_{0}}\right)} Z_{t}^{v}\left(x_{1_{0}}, x_{2_{0}} ; x_{1}, x_{2}\right) .
$$

Здесь $Z_{t}^{v}\left(x_{1_{0}}, x_{2_{0}} ; x_{1}, x_{2}\right)$ - фундаментальное решение уравнения Блоха

$$
\frac{\partial}{\partial t} Z_{t}^{v}\left(x_{1_{0}}, x_{2_{0}} ; x_{1}, x_{2}\right)=-\widehat{H}^{v} Z_{t}^{v}\left(x_{1_{0}}, x_{2_{0}} ; x_{1}, x_{2}\right)
$$

Отметим важный частный случай, когда спектр оператора Гамильтона $\widehat{H}^{v}$ дискретный, а $z_{-t}^{v}(x)$ отвечает функции основного состояния этого оператора. При этом происходит естественная перекалибровка оператора $\widehat{H}^{v}$, и в результате он аннулирует функцию основного состояния, а распределение Больцмана оказывается квадратом этой функции (см. [7], ср. также с [5]).

Отметим, что в силу полученной абсолютной непрерывности мер Винера и трансформированной меры (5), представляющей решение задачи об евклидовой квантовой частице фиксированной массы в произвольном заданном внешнем поле, можно сказать, что квантовая задача в евклидовом представлении порождается общей для всех этих задач микроструктурой пространства-времени винеровских процессов $\varphi(s)$ (или $x(s)$ ), по которым достаточно интегрировать в интеграле Винера (якобиан в (5) не обращается в нуль). Носителем меры Винера являются процессы $\varphi(s)$, являющиеся непрерывными, но не дифференцируемыми, и удовлетворяющие условию Гельдера-Липшица $|\varphi(s+\Delta s)-\varphi(s)| \leqslant A|\Delta s|^{1 / 2-\varepsilon}$, где $A>0, \varepsilon<1 / 2$ любые, так что носитель меры Винера локально компактен в $C$ (пространстве непрерывных функций) [5]. При этом указанная структура пространства-времени одинаково успешно определяется путем применения причинных функций Грина частиц фиксированной массы в различных полях (например, это может быть трехмерная свободная частица, трехмерный осциллятор или атом водорода). Таким образом, решение каждой конкретной задачи может интерпретироваться квантовый прибор, не возмущающий и не проектирующий, как классический прибор, но одинаково точно описывающий указанную пространственно-временную структуру. Вместе с тем переход от одного гамильтониана к другому в случае частицы с фиксированной массой представляет собой каноническое преобразование, вполне аналогичное соответствующему каноническому преобразованию в классической механике.

Таков метод построения на базе свободных частиц функций Грина взаимодействующих евклидовых квантовых частиц с использованием гладкости этих функций 
Грина при всех $t>0$ (за исключением лишь одного значения $t=0$, что, очевидно, не меняет сути дела).

Но для нас важно, что соотношению (9) (или (10)) может быть придан иной смысл, позволяющий получить те же результаты, если считать все функции Грина распределениями и при этом не использовать стохастические уравнения.

В случае нерелятивистской квантовой механики это независимый альтернативный путь; в случае релятивистской теории это, по-видимому, единственная возможность учесть внешнее электромагнитное поле и взаимодействие между электронами Дирака, так как все функции Грина в этом случае являются обобщенными функциями [8].

Итак, сначала укажем альтернативный метод учета взаимодействия между нерелятивистскими евклидовыми квантовыми частицами, а затем распространим его на релятивистский случай.

Воспользуемся тем, что фундаментальное решение уравнения Шредингера для свободной частицы (уравнение диффузии) известно:

$$
Z_{t}\left(x-x_{0}\right)=\frac{1}{\sqrt{\pi t}} \exp \left\{-\frac{\left(x-x_{0}\right)^{2}}{t}\right\}
$$

будучи распределением, оно удовлетворяет уравнению Чепмена-Колмогорова

$$
\int Z_{t}(\alpha) Z_{s}(\beta) \psi(\alpha+\beta) d \alpha d \beta=\int Z_{t+s}(\gamma) \psi(\gamma) d \gamma
$$

Покажем, что (11) определяет обобщенную вероятностную счетно-аддитивную меру на пространстве $S$ быстроубывающих функций [9]. После этого мы обнаружим абсолютную непрерывность друг по другу мер, отвечающих невзаимодействующим евклидовым квантовым частицам и частицам во внешних полях.

Отметим прежде всего, что (11) как функционал удовлетворяет принципу причинности, откуда следует, что

$$
\int\left[\prod_{j=1}^{n} Z_{\Delta t_{j}}\left(y_{j}\right)\right] \psi\left(\sum_{j=1}^{n} y_{j}\right) d y_{1} \ldots d y_{n}=\int Z_{t}(y) \psi(y) d y
$$

(здесь $0=t_{0} \leqslant t_{1} \leqslant \cdots \leqslant t_{n}=t, \Delta t_{j}=t_{j}-t_{j-1}$ ) для любой основной функции $\psi(y) \in Z[10]$. Покажем, что причинная функция (11) как функционал определяет плотности вероятности значений $A_{1}, \ldots, A_{k}$ линейных функционалов

$$
\int_{0}^{t} a_{l}(\tau) d x(\tau)=A_{l}, \quad l=1, \ldots, k,
$$

или, для начала, линейных функционалов

$$
\sum_{j=1}^{n} a_{l_{j}} \Delta x_{j}=A_{l}
$$

Для этого рассмотрим интеграл

$$
\int\left[\prod_{j=1}^{n} Z_{\Delta t_{j}}\left(\Delta x_{j}\right)\right] \Psi\left(\sum_{j=1}^{n} a_{1_{j}} \Delta x_{j}, \ldots, \sum_{j=1}^{n} a_{k_{j}} \Delta x_{j}\right) d x_{1} \ldots d x_{n},
$$


где $\Psi\left(A_{1}, \ldots, A_{k}\right) \in Z^{k}$. Запишем теперь эту аналитическую функцию через интеграл Фурье:

$$
\begin{aligned}
& \Psi\left(\sum_{j=1}^{n} a_{1_{j}} \Delta x_{j}, \ldots, \sum_{j=1}^{n} a_{k_{j}} \Delta x_{j}\right)= \\
& \quad=(2 \pi)^{-k} \int \exp \left\{-i \sum_{l=1}^{k} \sum_{j=1}^{n} a_{l_{j}} \Delta x_{j} p_{l}\right\} \Phi\left(p_{1}, \ldots, p_{k}\right) d p_{1} \ldots d p_{k} .
\end{aligned}
$$

Здесь $\Phi\left(p_{1}, \ldots, p_{k}\right) \in K^{k}-$ финитная функция $k$ переменных (см. [10]).

Подставим это разложение в (15), получим

$$
\int \prod_{j=1}^{n}\left[\int Z_{\Delta t_{j}}\left(\Delta x_{j}\right) \exp \left\{-i \sum_{l=1}^{k} a_{l_{j}} \Delta x_{j} p_{l}\right\} d \Delta x_{j}\right] \Phi\left(p_{1}, \ldots, p_{k}\right) d p_{1} \ldots d p_{k} .
$$

Внутренние интегралы известны, поэтому перепишем (15) как

$$
\int \exp \left\{-\frac{1}{4} \sum_{j=1}^{n}\left(\sum_{l=1}^{k} p_{j} a_{l_{j}}\right)^{2} \Delta t_{j}\right\} \Phi\left(p_{1}, \ldots, p_{k}\right) d p_{1} \ldots d p_{k} .
$$

Отсюда, устремляя все $\Delta t_{j}$ к нулю, получаем следующий образ Фурье обобщенной функции распределения значений функционалов (14):

$$
\exp \left\{-\frac{1}{4} \sum_{l, m=1}^{k} p_{l} p_{m} \int_{0}^{t} a_{l}(\tau) a_{m}(\tau) d \tau\right\}
$$

Эта обобщенная функция гауссова, и можно было бы найти функцию плотности вероятности распределения величин $A_{1}, \ldots, A_{k}$, однако мы этого делать не будем, так как для нас важно не само распределение, а его счетная аддитивность. Это свойство распределения следует из того, что обобщенная функция (17) положительно определена, поэтому по теореме Бохнера [9] определяет положительную (вероятностную) меру цилиндрических множеств как обобщенную функцию на пространстве $S$ быстроубывающих функций, причем в данном случае эта мера согласована и нормирована. Согласно теореме Минлоса [9] эта мера счетно-аддитивна.

Вернемся к тому факту, что произведение любой пары решений уравнений (1) и (2) удовлетворяет известному уравнению непрерывности в квантовой механике:

$$
\frac{\partial}{\partial t}\left[z_{-t}^{v}(x) Z_{t}^{v}\left(x_{0}, x\right)\right]-\frac{1}{4} \frac{\partial}{\partial x}\left[z_{-t}^{v}(x) \frac{\partial Z_{t}^{v}\left(x_{0}, x\right)}{\partial x}-\frac{\partial z_{-t}^{v}(x)}{\partial x} Z_{t}^{v}\left(x_{0}, x\right)\right]=0
$$

(это уравнение может быть тождественно переписано как уравнение Фоккера-Планка $(7)$ для $Z_{t}^{v}\left(x_{0}, x\right)$ при заданном $\left.z_{-t}^{v}(x)[7]\right)$.

Структура уравнения (18) позволяет применить вариант известной теоремы Хольмгрена-Гельфанда о связи обобщенного решения линейного дифференциального уравнения (1) с решением обратно сопряженного к нему уравнения (2) для основных функций [4]. 
В самом деле, в результате интегрирования уравнения непрерывности (18) по всему пространству обнаруживаем, что $\int z_{-t}^{v}(x) Z_{t}^{v}\left(x_{0}, x\right) d x$ на самом деле не зависит от времени $t$. Отсюда, переходя формально в этом интеграле к пределу $t \rightarrow 0$, получаем, что для фундаментального решения $Z_{t}^{v}\left(x_{0}, x\right)$ уравнения (1) и для любого решения $z_{-t}^{v}(x)$ уравнения (2) справедливо соотношение

$$
\int z_{-t}^{v}(x) Z_{t}^{v}\left(x_{0}, x\right) d x=z_{0}^{v}\left(x_{0}\right)
$$

так что $Z_{t}^{v}\left(x_{0}, x\right)$ как функционал действительно оказывается определенным на множестве решений уравнения (2) как на основных функциях [4]. Но уравнение (2) является обратно сопряженным по отношению к уравнению (1), так что совокупность его решений $z_{-t}^{v}(x)$ можно рассматривать как множество основных функций, на которых однозначно (а на части их и корректно) задано решение уравнения (1) как распределение. И это в самом деле оказывается справедливым, если учесть эллиптичность оператора Гамильтона $\widehat{H}^{v}$ в (2) [4].

Отметим также, что $z_{-t}^{v}(x)$ как функции от $x$ принадлежат пространствам типа $S$ (см. [10]).

Основные функции $z_{-t}^{v}$ удовлетворяют уравнению (2), поэтому из (19) следует, что распределение $Z_{t}^{v}\left(x_{0}, x_{t}\right)$ есть фундаментальное решение уравнения (1). В самом деле, вспоминая, что $t$ в распределении $Z_{t}^{v}\left(x_{0}, x_{t}\right)$ есть параметр, и дифференцируя (19) по $t$, имеем

$$
\int\left[\frac{\partial z_{-t}^{v}\left(x_{t}\right)}{\partial t} Z_{t}^{v}\left(x_{0}, x_{t}\right)+z_{-t}^{v}\left(x_{t}\right) \frac{\partial Z_{t}^{v}\left(x_{0}, x_{t}\right)}{\partial t}\right] d x_{t}=0,
$$

откуда, используя эрмитовость $\widehat{H}^{v}$, получаем

$$
\int z_{-t}^{v}\left(x_{t}\right)\left[\frac{\partial Z_{t}^{v}\left(x_{0}, x_{t}\right)}{\partial t}+\widehat{H}^{v} Z_{t}^{v}\left(x_{0}, x_{t}\right)\right] d x_{t}=0
$$

для любой удовлетворяющей (2) основной функции $z_{-t}^{v}(x)$. Таким образом, из (19) следует, что справедливо уравнение (1). Из (19), как отмечалось выше, следует

$$
\lim _{t \rightarrow 0} Z_{t}^{v}\left(x_{0}, x\right)=\delta\left(x-x_{0}\right)
$$

так что выполнение уравнения (19) при любом решении $z_{-t}^{v}(x)$ уравнения (2) действительно определяет фундаментальное решение $Z_{t}^{v}\left(x_{0}, x\right)$ уравнения (1) как распределение.

Отметим отличия в методах получения и в сути соотношений (9) и (19). С одной стороны, соотношение (9) определяет структуру фундаментального решения уравнения Фоккера-Планка $(7)$, причем $W_{t}\left(x_{0}, x_{t}\right)$ понимается как обычная функция и порождается одним конкретным решением уравнения (2). С другой стороны, (19) возникает, если интерпретировать уравнение $(2)$ как уравнение для $Z_{t}^{v}\left(x_{0}, x\right)$ как распределения. Последнее оказывается возможным лишь при учете того факта, что (19) справедливо для множества решений $z_{-t}^{v}(x)$ уравнения $(2)$, что и позволяет 
придать (19) смысл определения $Z_{t}^{v}\left(x_{0}, x\right)$ как функционала. При такой интерпретации исчезает необходимость рассматривать стохастические уравнения, лежащие в основании уравнения (9), так как (19) получается сразу из уравнения непрерывности (18). Именно в этом смысле (19) достигает той же цели, что и аппарат стохастических уравнений, при решении задачи о введении внешнего поля в нерелятивистской квантовой механике (об аналогичной задаче в релятивистской квантовой механике см. ниже).

Отметим также тесную связь этого подхода с обычным функциональным интегрированием. Для любого разбиения отрезка времени $[0, t]$ последовательными точками $t_{1}, t_{2}, \ldots, t_{n}=t$ справедливо равенство

$$
\int \ldots \int\left\{\prod_{j=1}^{n} \frac{z_{-t_{j}}^{v}\left(x_{j}\right)}{z_{-t_{j-1}}^{v}\left(x_{j-1}\right)} Z_{t_{j}-t_{j-1}}^{v}\left(x_{j-1}, x_{j}\right)\right\} d x_{1} \ldots d x_{n-1}=\frac{z_{-t}^{v}\left(x_{t}\right)}{z_{0}^{v}\left(x_{0}\right)} Z_{t}^{v}\left(x_{0}, x_{t}\right)
$$

что в предельном случае при $\max _{j} \Delta t_{j} \rightarrow 0$ очевидно эквивалентно винеровскому интегралу (8) и поясняет возникновение формулы (9).

Вместе с тем имеет место взаимосвязь основных функций $z_{-t}^{v}(x)$ и $z_{-t}^{v^{\prime}}(x)$, отвечающих операторам Гамильтона с разными потенциалами $V(x)$ и $V^{\prime}(x)$ (что при ранее рассмотренном подходе описывается различными стохастическими уравнениями (3)). Эти две основные функции связаны непрерывным линейным отображением, так как в силу произвольности начальных данных в обратно сопряженных уравнениях для основных функций всегда можно положить $z_{0}^{v}\left(x_{0}\right)=z_{0}^{v^{\prime}}\left(x_{0}\right)$. Такая связь основных функций обеспечивает связь мер цилиндрических множеств, отвечающих разным операторам Гамильтона (с разными $V(x)$ и $V^{\prime}(x)$ ), как индуцированных мер (см. [9]). Эта связь основных функций, очевидно, играет ту же роль, что и отличие от нуля знаменателя Фредгольма в интеграле Винера (5), обеспечивая существование фундаментального решения уравнения (1) $Z_{t}^{v}\left(x_{0}, x\right)$ как распределения для различных $V(x)$, если это выполняется лишь для одного из таких потенциалов (нулевого, отвечающего счетно-аддитивной мере Винера). При этом возникает связь этих фундаментальных решений как абсолютно непрерывных обобщенных мер, чем и завершается построение альтернативного методу стохастических уравнений метода, базирующегося на теории распределений.

Таким образом, в нерелятивистской квантовой теории переход от свободных квантовых частиц к взаимодействующим частицам мы можем осуществить двумя путями: либо рассматривая фундаментальные решения как обычные функции (используя тот факт, что они становятся обобщенными лишь при $t \rightarrow 0)$, и тогда можно использовать аппарат стохастических уравнений; либо рассматривая их как распределения, которые задаются решениями соответствующих обратно сопряженных уравнений как основными функциями. При этом стохастические уравнения оказываются ненужными, а переход от свободной квантовой частицы к частице во внешнем поле на языке обобщенных функций можно интерпретировать как переход от основных функций, на которых однозначно (и корректно) задано фундаментальное решение уравнения диффузии как распределение (этим уравнением является уравнение диффузии вспять во времени, подробности см. в [4]), к основным функциям, 
на которых однозначно (и корректно) задано фундаментальное решение уравнения (1) как распределение (теорема Хольмгрена-Гельфанда). Как отмечалось, такими основными пространствами являются пространства типа $S$ (см. [4], [11]). Тем же способом можно точно учесть произвольное взаимодействие между квантовыми частицами.

Напомним еще раз, что замена переменных в интеграле Винера приводит к введению внешнего поля или к учету взаимодействия частиц в евклидовой квантовой механике. Вместе с тем аппарат изменения множества основных функций, отвечающего изменению внешнего поля (или отвечающего заданному взаимодействию между частицами), не опирается на специфическую зависимость винеровской меры от траектории брауновской частицы и на гладкость траекторий, по которым достаточно интегрировать в винеровском интеграле. Однако, используя этот аппарат, мы достигаем поставленной цели, если понимаем решение как распределение. При этом аналогом пространства винеровских траекторий является единое для всех рассматриваемых гамильтонианов пространство основных функций $S$. Отметим также, что сохраняется указанная выше интерпретация как канонического преобразования процедуры построения функции Грина.

\section{3. УРАВНЕНИЕ ДИРАКА}

Перенесем теперь метод, изложенный выше, на уравнение Дирака. В этом случае особую роль играют евклидовы решения уравнений Дирака для нейтрино и антинейтрино, причем нейтрино отвечают в представлении, аналогичном представлению Фолди-Вотхойзена [12], два одинаковых процесса с переходной вероятностью [8]

$$
C_{t}(x)=\frac{t}{\pi^{2}\left(t^{2}+r^{2}\right)^{2}}, \quad r=|x| .
$$

Как оказалось, $C_{t}(x)$ является переходной вероятностью процесса Коши, который отвечает пространственному закону распределения Коши [13]. Каждое такое решение уравнений Дирака представляет собой обобщенную вероятностную счетноаддитивную меру на основном пространстве $S$ [8].

Как было показано в той же работе [8], в этом случае антинейтрино отвечают также две одинаковые причинные функции $B_{t}(x)$, являющиеся, как и $(22)$, распределениями на $Z$, т.е. аналитическими функционалами. Поэтому, в отличие от нерелятивистской квантовой механики, фундаментальные решения в случае уравнений Дирака можно рассматривать только как распределения.

Ясно, что функционал $B_{t}(x)$, образ Фурье которого есть

$$
\widetilde{B}_{t}(p)=e^{t \rho}
$$

не является знакоопределенной функцией. Покажем, что функционал $B_{t}(x)$ есть функционал со слабо ограниченной вариацией [4], и поэтому (несмотря на комплекснозначность) (23), как и (22), будучи нормированным распределением, также определяет счетно-аддитивную обобщенную меру на $S$. 
В самом деле, оценим слабую вариацию функционала (23)

$\operatorname{Var} B_{t}(x)=\sum_{j=1}^{n}\left|\left(B_{t_{j}}(x), \psi(x)\right)-\left(B_{t_{j-1}}(x), \psi(x)\right)\right|, \quad\left(B_{t}(x), \psi(x)\right)=\int B_{t}(x) \psi^{*}(x) d x ;$

здесь $0=t_{0} \leqslant t_{1} \leqslant \cdots \leqslant t_{n}=t$ - любое разбиение отрезка $[0, t]$ а основная функция $\psi(x)$ принадлежит $Z$, т.е. является целой аналитической функцией первого порядка роста (см. [8], [10], [11]). Воспользуемся формулой Парсеваля и перейдем в (24) к импульсному представлению; в результате получим

$$
\operatorname{Var} B_{t}(x)=\frac{1}{(2 \pi)^{3}} \sum_{j=1}^{n}\left|\left(e^{t_{j} \rho}, \varphi(p)\right)-\left(e^{t_{j-1} \rho}, \varphi(p)\right)\right|,
$$

где $\varphi(p) \in K-$ финитная основная функция. Легко видеть, что

$$
\frac{1}{(2 \pi)^{3}} \sum_{j=1}^{n}\left|\left(\left[e^{\left(t_{j}-t_{j-1}\right) \rho}-1\right] e^{t_{j} \rho}, \varphi(p)\right)\right| \leqslant \frac{t}{(2 \pi)^{3}}\left(\rho e^{2 t \rho}, \varphi(p)\right)<\infty
$$

так что вариация (24) не зависит от разбиения отрезка $[0, t]$. Из (26) следует, что функционал $B_{t}(x)$ имеет слабо ограниченную вариацию.

Функционал $B_{t}(x)$ является причинной функцией Грина и потому определяет согласованную нормированную (см. (23)) меру цилиндрических множеств с произвольными конечномерными основаниями (см. [9]; конкретное построение этих мер точно такое же, как в [8]). Вариация $B_{t}(x)$ слабо ограничена, и $B_{t}(x)$ как аналитический функционал может быть продолжен на пространство $S$ быстроубывающих функций. Отсюда в силу простой модификации теоремы Минлоса [9] следует возможность продолжения $B_{t}(x)$ на тело борелевских множеств, таким образом, $B_{t}(x)-$ счетно-аддитивная комплексная обобщенная мера.

Отметим также, что переходная вероятность процесса Коши порождает обобщенную счетно-аддитивную вероятностную меру, для которой мера непрерывных функций равна нулю (траектории, по которым движутся евклидовы нейтрино, с вероятностью единица каждое мгновение испытывают разрыв, см. [14]).

Поэтому, несмотря на то что переходная вероятность процесса Коши $C_{t}(x)$ непрерывно зависит от пространственных переменных (иначе говоря, это функционал типа функции), применение метода стохастических уравнений в его обычной форме даже для одного процесса Коши невозможно.

Однако эффективной оказывается конструкция Хольмгрена-Гельфанда, определяющая причинные функции Грина нейтрино, свободного электрона и электрона в поле как распределения. Особенно важно, что при этом обнаруживаются взаимосвязи функций Грина, отвечающих частице в различных полях в релятивистской теории, если эти функции рассматривать как распределения.

Траектории нейтрино с вероятностью единица в каждый момент времени представляют собой разрывные функции, так что эти частицы чисто кинематически имеют возможность оказаться с другой стороны площадки, не пересекая ее, что, видимо, способствует пониманию особых проникающих свойств нейтрино. 
Напомним уравнения Дирака для электрона во внешнем электростатическом поле с потенциалом $\varphi(x)[12]$ :

$$
\gamma^{0}\left[i \frac{\partial}{\partial t}-e \varphi(x)\right] d_{t}^{m e}(x)+(\gamma, i \nabla) d_{t}^{m e}(x)-m d_{t}^{m e}(x)=0 .
$$

Здесь $e$ - заряд электрона, $m$ - его масса, скорость света $C$ и постоянная Планка $\hbar$ положены равными единице, $r$ - модуль вектора $x,(\gamma, \nabla)=\sum_{j=1}^{3} \gamma^{j} \frac{\partial}{\partial x^{j}}, \gamma^{\mu}$ - матрицы Дирака, $d_{t}^{m e}(x)$ - столбец. Это уравнение в евклидовом варианте приводится к виду

$$
\frac{\partial}{\partial t} d_{-i t}^{m e}(x)=\widehat{H}^{m e} d_{-i t}^{m e}(x),
$$

где $\widehat{H}^{m e}=\gamma^{0}(\gamma,-i \nabla)+\gamma^{0} m+\operatorname{Ie\varphi }(x)$.

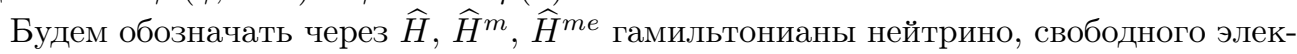
трона и электрона в поле, соответственно, те же верхние индексы мы будем использовать для обозначения решений уравнений Дирака с соответствующими гамильтонианами.

Пусть $D_{t}^{m e}\left(x_{0}, x\right)$ - фундаментальное решение (матрица) уравнения (27), $D_{-i t}^{m e}\left(x_{0}, x\right)$ - фундаментальное решение $(28)$, а строка $d_{-i t}^{m e}(x)$ - решение обратно сопряженного к (28) уравнения [4]

$$
-\frac{\partial}{\partial t} d_{-i t}^{m e^{*}}(x)=d_{-i t}^{m e^{*}}(x) \widehat{H}^{m e^{*}}
$$

где $*$ означает эрмитово сопряжение, так что выполняется уравнение непрерывности

$$
\frac{\partial}{\partial t}\left[d_{-i t}^{m e^{*}}(x) D_{-i t}^{m e}\left(x_{0}, x\right)\right]=\left(-i \nabla, d_{-i t}^{m e^{*}}(x) \gamma^{0} \gamma D_{-i t}^{m e}\left(x_{0}, x\right)\right) .
$$

Интегрируя это уравнение по пространственным переменным, получаем

$$
\frac{\partial}{\partial t} \int d_{-i t}^{m e^{*}}(x) D_{-i t}^{m e}\left(x_{0}, x\right) d x=0 .
$$

Так как это уравнение справедливо для любого решения $d_{i t}^{m e^{*}}(x)$ уравнения (29), мы имеем следующее уравнение для фундаментального решения $D_{-i t}^{m e}\left(x_{0}, x\right)$ как для обобщенной функции:

$$
\int d_{-i t}^{m e^{*}}(x) D_{-i t}^{m e}\left(x_{0}, x\right) d x=d_{0}^{m e^{*}}\left(x_{0}\right),
$$

которое однозначно определяет $D_{-i t}^{m e}\left(x_{0}, x\right)$ согласно теореме Хольмгрена-Гельфанда (основные функции $d_{-i t}^{m e^{*}}(x)$ удовлетворяют эллиптической системе дифференциальных уравнений (29)). В самом деле, выполняя дифференцирование в (31) и используя (29), получаем

$$
\int d_{-i t}^{m e^{*}}(x)\left[\frac{\partial}{\partial t} D_{-i t}^{m e}\left(x_{0}, x\right)-\widehat{H}^{m e} D_{-i t}^{m e}\left(x_{0}, x\right)\right] d x=0
$$

для любого решения $d_{-i t}^{m e^{*}}(x)$ уравнения (29) как для основной функции; поэтому по теореме Хольмгрена-Гельфанда соотношение (33) однозначно определяет $D_{-i t}^{m e}\left(x_{0}, x\right)$ 
как распределение. Отметим полную аналогию с нерелятивистским случаем, см. уравнение (20).

В случае евклидова нейтрино образ Фурье фундаментального решения имеет вид $\widetilde{D}_{-i t}(p)=\widetilde{T}(p) e^{-t \gamma^{0} \rho} \widetilde{T}(p)$, где $\rho-$ модуль вектора $p, p_{\text {e }}$ - его единичный вектор, а $\widetilde{T}(p)=\gamma^{0}\left[\left(\gamma, p_{\mathrm{e}}\right)+I\right] / \sqrt{2}$ (см. [8]). Поэтому с учетом перечисленных выше свойств процесса Коши $C_{t}(x)$ и процесса $B_{t}(x)$ решение $D_{-i t}(x)$ также представляет собой обобщенную матричнозначную комплексную нормированную счетноаддитивную меру.

Сопоставим теперь уравнения, содержащие фундаментальные решения как распределения, для нейтрино, свободного электрона и электрона в электростатическом поле:

$$
\begin{aligned}
& \int d_{-i t}^{*}(x) D_{-i t}\left(x-x_{0}\right) d x=d_{0}^{*}\left(x_{0}\right), \\
& \int d_{-i t}^{m^{*}}(x) D_{-i t}^{m}\left(x-x_{0}\right) d x=d_{0}^{m^{*}}\left(x_{0}\right), \\
& \int d_{-i t}^{m e^{*}}(x) D_{-i t}^{m e}\left(x_{0}, x\right) d x=d_{0}^{m e^{*}}\left(x_{0}\right),
\end{aligned}
$$

где $d_{-i t}^{*}(x), d_{-i t}^{m^{*}}(x), d_{-i t}^{m e^{*}}(x)$ - произвольные решения соответствующих уравнений типа (29). Эти решения, играющие роль основных функций при определении соответствующих фундаментальных решений как распределений, связаны линейным отображением, что следует из непрерывной зависимости от начальных данных решений уравнений (29) и возможности отождествить начальные данные для этих уравнений. Поэтому, как и в нерелятивистском случае, возникает естественная взаимосвязь мер цилиндрических множеств, отвечающих $D_{-i t}\left(x-x_{0}\right), D_{-i t}^{m}\left(x-x_{0}\right)$ и $D_{-i t}^{m e}\left(x_{0}, x\right)$ как индуцированных мер (см. [9]). Вместе с тем, так как одна из этих мер (отвечающая нейтрино) счетно-аддитивна, все эти меры счетно-аддитивны и абсолютно непрерывны. Отметим, что в [8] эта связь фундаментальных решений нейтрино и электрона была обнаружена иначе. Напомним также, что меры, отвечающие различным массам евклидовых частиц в нерелятивистской теории, не абсолютно непрерывны друг по другу.

Отметим важное следствие из структуры уравнений Дирака, являющееся характерным именно для релятивистской квантовой механики: существование кинематической зависимости движений по ортогональным направлениям в решениях уравнения Дирака. Покажем, что, пренебрегая этим эффектом, мы придем к нарушению уравнения непрерывности в конкретной задаче, причем этот эффект исчезает уже в квазиклассическом приближении.

Рассмотрим сначала вопрос об иллюстрации известного парадокса Клейна (об уравнении Дирака для электрона во внешнем электростатическом поле см., например, [12]), состоящего в том, что согласно [12] нарушается уравнение непрерывности при рассеивании частицы Дирака на прямоугольном барьере конечной высоты. Хорошо известно, что аналогичная задача для уравнения Шредингера не приводит к нарушению уравнения непрерывности (см., например, [15]). 
Напомним, что импульсное представление фундаментального решения уравнения Шредингера в случае свободной частицы есть

$$
\exp \left\{\frac{i t}{4} \rho^{2}\right\}
$$

а в случае уравнения Дирака -

$$
\exp \left\{i t \gamma^{0}[(p, \gamma)+I m]\right\}, \quad(p, \gamma)=\sum_{j=1}^{3} p_{j} \gamma^{j} .
$$

Принципиальное различие этих решений заключается в том, что в случае уравнения Шредингера

$$
\exp \left\{\frac{i t}{4} \rho^{2}\right\}=\prod_{j=1}^{3} \exp \left\{\frac{i t}{4} p_{j}^{2}\right\}
$$

следовательно, возможно движение вдоль одной из осей (например, $x^{1}$ ) при наличии произвольного движения в ортогональной плоскости $\left(x^{2}, x^{3}\right)$, причем эти движения совершенно независимы.

С другой стороны,

$$
\exp \left\{i t \gamma^{0}[(p, \gamma)+I m]\right\} \neq \exp \left\{i t \gamma^{0} m\right\} \prod_{j=1}^{3} \exp \left\{i t \gamma^{0} p_{j} \gamma^{j}\right\}
$$

из-за некоммутативности матриц Дирака, так что в релятивистской квантовой механике, в отличие от релятивистской классической механики, кинематически взаимосвязаны движения по взаимно ортогональным направлениям. Поэтому в случае релятивистской квантовой теории в отличие от уравнения Шредингера принципиально невозможно рассматривать одномерное движение (например, вдоль оси $x^{1}$ ) без учета движения в плоскости, ортогональной к этому движению (в частности, в случае плоской волны). Только после интегрирования по переменным, определяющим движение в этой плоскости (в плоскости $\left(x^{2} x^{3}\right)$ ), можно получить решение уравнения Дирака, зависящее лишь от $x^{1}$. В импульсном представлении в результате этого интегрирования получается $\exp \left\{i t \gamma^{0}\left[p_{1} \gamma^{1}+I m\right]\right\}$.

Поэтому проблема нарушения релятивистского уравнения непрерывности отпадает сама собой: она возникла из-за пренебрежения движением в плоскости, ортогональной волновому вектору падающей волны. Заметим, что учесть это движение при поиске решения в виде плоской волны принципиально невозможно. Понятно, что приведенные рассуждения не позволяют сомневаться в существовании самого парадокса Клейна.

В заключение покажем, что уже при квазиклассическом приближении квантовых релятивистских задач исчезает отмеченная выше кинематическая взаимосвязь движений по ортогональным направлениям.

В случае отсутствия поля обобщенное (фундаментальное) решение $D_{-i t}^{m}\left(x-x_{0}\right)$ уравнения Дирака определяется из соотношения

$$
\int d_{-i t}^{m^{*}}(x)\left[i \frac{\partial}{\partial t} D_{-i t}^{m}\left(x-x_{0}\right)-\widehat{H}^{m} D_{-i t}^{m}\left(x-x_{0}\right)\right] d x=0 .
$$


Воспользовавшись равенством Парсеваля, найдем образ Фурье обобщенной функции в квадратных скобках в (39). В результате получим

$$
\int \tilde{d}_{-i t}^{m^{*}}(p)\left\{i \frac{\partial}{\partial t} \widetilde{D}_{-i t}^{m}(p)-\left[\gamma^{0}(\gamma, p)+\gamma^{0} m\right] \widetilde{D}_{-i t}^{m}(p)\right\} d p=0
$$

Так как $\gamma^{0}(\gamma, p)+\gamma^{0} m-$ эрмитова матрица, существует оператор $\widetilde{T}^{m}(p)$, приводящий ее к диагональному виду. Легко видеть, что он является эрмитовым и унитарным оператором,

$$
\widetilde{T}^{m}(p)=\widetilde{T}^{m^{-1}}(p)=\gamma^{0} \frac{(\gamma, p)+I\left(m+\sqrt{m^{2}+\rho^{2}}\right)}{\left[2 \sqrt{m^{2}+\rho^{2}}\left(m+\sqrt{m^{2}+\rho^{2}}\right)\right]^{1 / 2}},
$$

так что

$$
\gamma^{0}(\gamma, p)+\gamma^{0} m=\widetilde{T}^{m}(p) \gamma^{0} \sqrt{m^{2}+\rho^{2}} \widetilde{T}^{m}(p)
$$

Заметим, что

$$
\lim _{m \rightarrow \infty} \widetilde{T}^{m}(p)=\gamma^{0}, \quad \lim _{m \rightarrow 0} \widetilde{T}^{m}(p)=\widetilde{T}(p) .
$$

Поэтому, действуя $\widetilde{T}^{m}(p)$ справа на (40), имеем

$$
\int \tilde{f}_{-i t}^{m^{*}}(p)\left\{i \frac{\partial}{\partial t} \widetilde{F}_{-i t}^{m}(p)-\gamma^{0} \sqrt{m^{2}+\rho^{2}} \widetilde{F}_{-i t}^{m}(p)\right\} d p=0,
$$

где $\widetilde{F}_{t}^{m}(p)$ и $\tilde{f}_{-i t}^{m^{*}}(p)$ - представления Фолди-Вотхойзена фундаментального решения $\widetilde{D}_{t}^{m}(p)$ и $\tilde{d}_{-i t}^{m^{*}}(p)$, соответственно, $\gamma^{0} \sqrt{m^{2}+\rho^{2}}-$ представление гамильтониана, а $\widetilde{T}^{m}(p)$ - преобразование Фолди-Вотхойзена [12].

Воспользуемся теперь тем, что прообраз абсолютно интегрируемой функции $\exp \left\{\tau \sqrt{m^{2}+p^{2}}\right\}$ при $\operatorname{Re} \tau<0$ известен [16], он задается как

$$
-\frac{\tau m}{2 \pi^{2} r} \frac{\partial}{\partial r} \frac{K_{1}\left(m \sqrt{\tau^{2}+r^{2}}\right)}{\sqrt{\tau^{2}+r^{2}}},
$$

где $K_{1}(z)$ - функция Ханкеля от чисто мнимого аргумента.

Восстанавливая физическое содержание $m$ и $t$ и используя известное асимптотическое представление $K_{1}(z)$ [17], получаем известную квазиклассическую аппроксимацию фундаментального решения уравнения Дирака для свободного электрона [18]:

$$
-I \sqrt{\frac{m C}{i(2 \pi)^{3} \hbar}} \frac{C t}{r} \frac{\partial}{\partial r}\left[\exp \left\{\frac{i}{\hbar} m C S\right\} S^{3 / 2}\right],
$$

где $C$ - скорость света, $\hbar$ - постоянная Планка, $S=\sqrt{(C t)^{2}-r^{2}}-$ интервал.

Важно отметить, что в отличие от нерелятивистского случая, когда квантование осуществляется просто путем отказа от принципа наименьшего действия (формула Каца-Фейнмана [1], [5]), при квантовании релятивистской частицы возникает уравнение, фундаментальное решение которого есть обобщенная функция, уже поэтому не имеющая структуры интеграла по путям. Таким образом, возникновение решения этого уравнения невозможно интерпретировать просто как следствие отказа 
от принципа наименьшего действия. Вместе с тем обнаруженная выше фундаментальная кинематическая зависимость движений по ортогональным направлениям, являясь отличительным признаком квантовой релятивистской теории, утрачивается уже в квазиклассическом приближении, как это следует из (46). При этом структура квазиклассического приближения оказывается точно такой же, как и в нерелятивистском случае.

\section{Список литературы}

[1] R. P. Feynman, Rev. Mod. Phys., 20:2 (1948), 367.

[2] Н.Н. Боголюбов, Д. В. Ширков, Введение в теорию квантованных полей, Наука, М., 1976.

[3] В. С. Владимиров, Обобщенные функиии в математической физике, Наука, М., 1979.

[4] И. М. Гельфанд, Г. Е. Шилов, Обобщенные функиии. Вып. 3. Некоторые вопросы теории дифференциальных уравнений, Физматгиз, М., 1958.

[5] Дж. Глимм, А. Джаффе, Математические методы квантовой физики. Подход с использованием функииональных интегралов, Мир, М., 1984.

[6] А.А. Бейлинсон, ДАН СССР, 128:5 (1959), 876.

[7] A. A. Beilinson, "On connection between Fokker-Colmogorov and Bloch-Schrödinger equations and Kac-Feynman formula", Proc. ICM-82, Short commun., Sec. 13, Warsaw, 1983, 13.

[8] А. А. Бейлинсон, ТМФ, 145:2 (2005), 157.

[9] И. М. Гельфанд, Н.Я. Виленкин, Обобщенные функиии. Bып. 4. Некоторые применения гармонического анализа. Оснащенные гильбертовы пространства, Физматгиз, M., 1961.

[10] И. М. Гельфанд, Г. Е. Шилов, Обобщенные функиии. Вып. 1. Обобщеннъе функиии и действия над ними, Физматгиз, М., 1958.

[11] И. М. Гельфанд, Г. Е. Шилов, Обобщенные функиии. Вып. 2. Пространства основных и обобщенных функиий, Физматгиз, М., 1958.

[12] К. Ициксон, Ж.-Б. Зюбер, Квантовая теория поля, т. 1, Мир, М., 1984.

[13] A. L. Cauchy, C. R. Acad. Sci., 37 (1853), 198.

[14] М. Кац, Несколько веролтностных задач физики и математики, Наука, М., 1967.

[15] Л.Д. Ландау, Е.М. Лифшиц, Квантовая механика. Нерелятивистская теория, ОГИЗ, М., 1948.

[16] В.П. Маслов, М.В. Федорюк, Квазиклассическое приближение для уравнений квантовой механики, Наука, М., 1976.

[17] Г. Бейтмен, А. Эрдейи, Таблицы интегралъных преобразований. Т. 2. Преобразования Бесселя. Интегралы от специальных функций, Наука, М., 1970.

[18] И.С. Градштейн, И.М. Рыжик, Таблицы интегралов, сумм, рядов и произведений, Физматгиз, М., 1963.

Поступила в редакцию 13.06.2006, после доработки 21.08.2006 